\title{
An Evaluation to the Effects of Correlation on Pseudo Noise Sequences
}

\author{
Nirmalya Chandra ${ }^{1}$, Sandeep Dey ${ }^{2}$, Sweta Shankar ${ }^{3}$, Roushan Kumar ${ }^{4}$ \\ Assistant Professor, Birbhum Institute of Engineering and Technology, Suri, Birbhum ${ }^{1}$ \\ Students, Birbhum Institute of Engineering and Technology, Suri, Birbhum ${ }^{2,3,4}$
}

\begin{abstract}
A PN sequence is a reference signal simulating the random samples of discrete digital information and generates the samples in a specific manner so that it follows the correlation property. A correlation and maximal length sequence of pseudo-random signals is the basis of spread spectrum communication systems intern CDMA. In this paper we examine a comparative study of PN sequence with theoretically measured correlation function at different value of total sequence.
\end{abstract}

Keywords: PN sequence, Autocorrelation Sequence, Cross-correlation Sequence, Spread Spectrum Modulation, LFSR circuit analysis.

\section{INTRODUCTION}

The pseudorandom noise (PN) sequences are a series of 1 's and 0's which lack any definite pattern, and look statistically independent and uniformly distributed. The sequences are deterministic, but exhibit noise properties similar to randomness. The time waveform generated from the PN sequences also seem like random noise. Thus the pseudo-random or pseudo-noise sequence is a noise-like spreading code.

A Pseudo random binary sequence is a semi-random sequence in the sense if appears random within the sequence length, fulfilling the needs of randomness, but the entire sequence repeats indefinitely. The paper [1] elaborates about various methods of generation of PN sequences, few methods discussed in this paper are seriesparallel method for high speed generation, avoiding zero states, shift registers with feedback, chaotic patterns generation. A detailed description is done here along with comparative analysis of LFSRs (Linear Feedback Shift Registers), Gold sequence, Kasami sequence is discussed in paper [2].

The series-parallel method of generating PN sequences at high speed with low speed devices, which is of interest to hardware designers is discussed in paper [3]. Applications demand [4] that the data appear random to the channel but be predictable to the user. In this scenario correlation is a measure of comparison between two data sequence which will be analyzed in this paper.

Through this paper we have tried to approach and explore the correlation properties of $\mathrm{PN}$ sequence. Correlation is a measure of similarity between two sequences. A good correlation property and large linear period of pseudorandom sequence is the basis of spread spectrum communication systems intern CDMA (code division multiple access). The autocorrelation property of PN sequences can be used for synchronization. Correlation is used at the receiver to acquire the PN sequence and decode the data.

\section{BLOCK DIAGRAM}

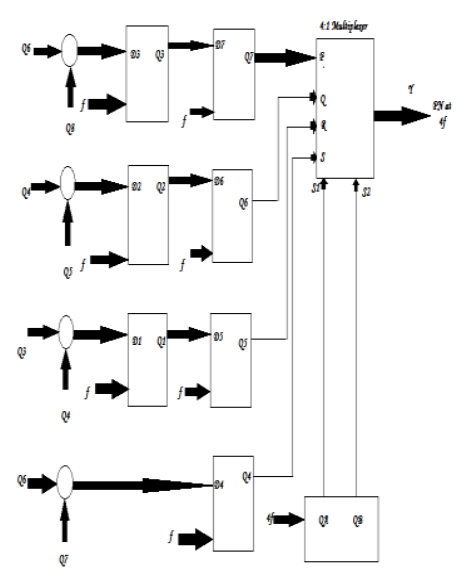

Figure 1 : Series-Parallel Generation of PN Sequence

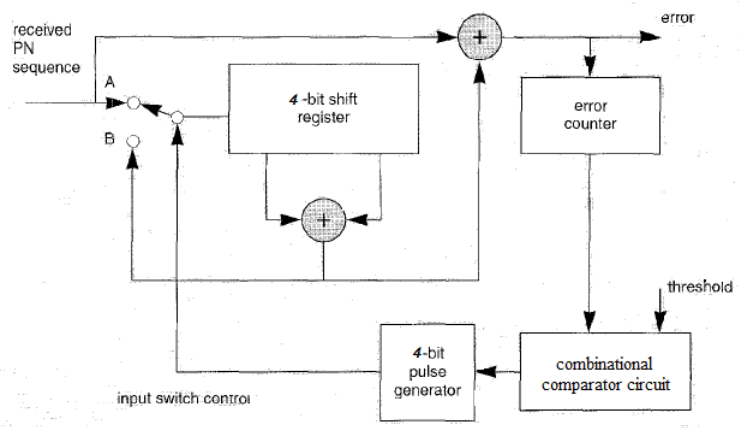

Figure 2: Acquisition of PN Sequence

\section{CONCEPT OF CORRELATION}

Correlation between any two phenomena implies the closeness with which they correspond in behavior and appearance

The primary objective of defining and computing correlation function between two sequences is to measure the extent to which the two sequences are similar, which is very useful if the sequences of interest are random in nature. 
The correlation of two sequences is divided into two :-

1. Cross-correlation ; 2. Auto-correlation

\section{A. CROSS CORRELATION :}

In signal processing, cross-correlation is a measure of similarity of two waveforms as a function of a time-lag applied to one of them.

This is also known as a sliding product or sliding innerproduct. It is commonly used for searching a long signal for a shorter, known feature. It has applications in pattern recognition, single particle analysis, electron tomography, averaging, and neurophysiology. cryptanalysis

For continuous functions $f$ and $g$, the cross-correlation is defined as:

$$
(f \star g)(\tau) \stackrel{\text { def }}{=} \int_{-\infty}^{\infty} f^{*}(t) g(t+\tau) d t,
$$

Where $f^{*}$ denotes the complex conjugate of $f$ and

$\tau$ is the time lag.

Similarly, for discrete functions, the cross-correlation is defined as:

$$
(f \star g)[n] \stackrel{\text { def }}{=} \sum_{m=-\infty}^{\infty} f^{*}[m] g[m+n] \text {. }
$$

\section{B. AUTO-CORRELATION :}

The auto-correlation of a sequence is the special case of cross correlation. The auto-correlation of a sequence is correlation of a sequence with itself.

The autocorrelation function $R(\mathrm{x})$ provides a measure of how closely the signal matches a copy of itself as the copy is shifted units in time. The variable plays the role of a scanning or searching parameter. $R(\mathrm{x})$ is not a function of time; it is only a function of the time difference between the waveform and its shifted copy.

Autocorrelation, also known as serial correlation, is the cross-correlation of a signal with itself. Informally, it is the similarity between observations as a function of the time lag between them. It is a mathematical tool for finding repeating patterns, such as the presence of a periodic signal obscured by noise, or identifying the missing fundamental frequency in a signal implied by its harmonic frequencies. It is often used in signal processing for analyzing functions or series of values, such as time domain signals. The auto correlation of a sequence $\mathrm{y}(\mathrm{n})[5]$ is defined by :

$R_{y y}(l)=\lim _{N \rightarrow \infty} \frac{1}{N} \sum_{n=0}^{N-1} y(n) \bar{y}(n-l)$.

Visual Comparison Study between the Correlation and Convolution :-

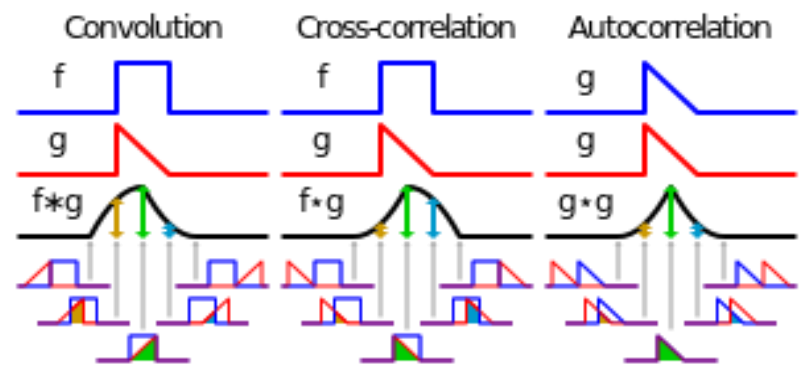

Figure 3 : Visual comparison of Convolution, CrossCorrelation and Autocorrelation [ Image from http://commons.wikimedia.org/wiki/File:Comparison_con volution_correlation.svg ]

\section{PN SEQUENCE GENERATION}

The PN sequences are created to determine and control the spreading pattern of the signal across the allotted bandwidth. The generation of PN sequences for spread spectrum applications is a topic that has received considerable attention in the technical literature.

Linear Feedback Shift Registers sequence through $\left(2^{n}-\right.$ 1) states, where $n$ is the number of registers in the LFSR. At each clock edge, the contents of the registers are shifted right by one position. There is feedback from predefined registers or taps to the left most register through an exclusive-NOR (XNOR) or an exclusive-OR (XOR) gate. A value of all "1"s is illegal in the case of a XNOR feedback. A count of all "0"s is illegal for an XOR feedback. This state is illegal because the counter would remain locked-up in this state. The LFSR in this application note is implemented with XNOR feedback. A 4-bit LFSR sequences through $\left(2^{4}-1\right)=15$ states (the state 1111 is in the lock-up/illegal state).

Since an LFSR has a finite set of states, the sequence of states eventually repeats. The number of states which appear before the first state repeats (and the sequence begins again) is called the period. Clearly, an LFSR with period $n$ can be used to count from 0 to $n-1$ : choose an arbitrary initial state giving rise to a sequence of period $n$, label this initial state " 0 ", and label every succeeding state by one more than the label of its predecessor.

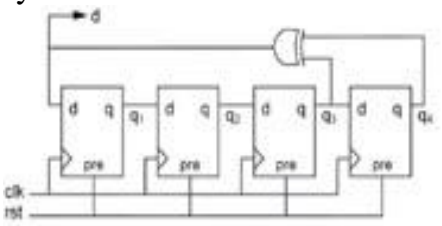

(a)

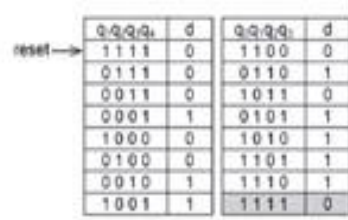

(b) (c)

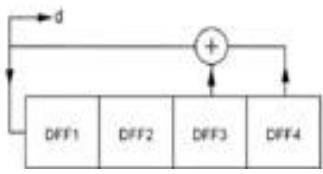

Figure 4 : (a) A 4-bit PN generator (b) The pattern of PN sequence generated from the LFSR. (c) LFSR structure.

\section{CORRELATION IN PN SEQUENCES}

The spread spectrum is made using a short periodic pseudo-random sequence with good correlation properties. The correlation properties of sequences are used to detect and synchronize the communication. To detect and 
recover such a message, the frequency of communication must be known, and then correlation test with each pseudo-random sequence must be used to find the good sequence and the synchronization

Mathematically, the correlation between two sequences $x$ $(k)$ and $y(k)$ as a function of the time delay $m$ is expressed as [7] :

$$
R(m)_{x y}=\sum_{k=0}^{L-1} x(k) y(k+m)
$$

For a digital bit stream the above equation can be interpreted in a simple way. Here $x(k)$ and $y(k)$, being digital bits, have binary values ' 0 ' or ' 1 '. The product, therefore, is simply the modulo- 2 addition. This is equivalent to exclusive NOR (XNOR) operation. Hence, the correlation of two serial bit sequences, such as PN sequences, is obtained over a length $L$ by comparing the two binary sequences bit-by-bit using XNOR gates, and counting the places they match. Thus, if the two bits are identical the XNOR produces a ' 1 ' at the output, otherwise a ' 0 '. The correlation is then obtained by counting all the ' 1 's and dividing the sum by the total number of bits, $L$, in the sequence, or the part of the sequence over which the correlation is calculated. The correlation equation for the digital bit sequence can thus be written as:

$R(m)=$ (number of agreements/total no. of bits)

In some literature the numerator in the above equation is expressed as the difference of the number of agreements and the number of disagreements

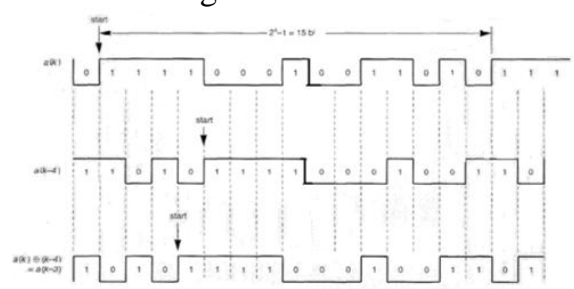

Figure 5 : Addition of PN sequence

However, eqn.(5) will be used here because it agrees with the general correlation eqn. 1, and also because the implementation is straight- forward as there is no need to form the difference of two sums. The difference in the results using the two definitions is only in the bias obtained in the correlation for all time lags $m$, where $m \neq 0$. To find the correlation over the entire sequence length one of the sequences is fixed while the other is shifted, and the correlation is obtained at different positions.

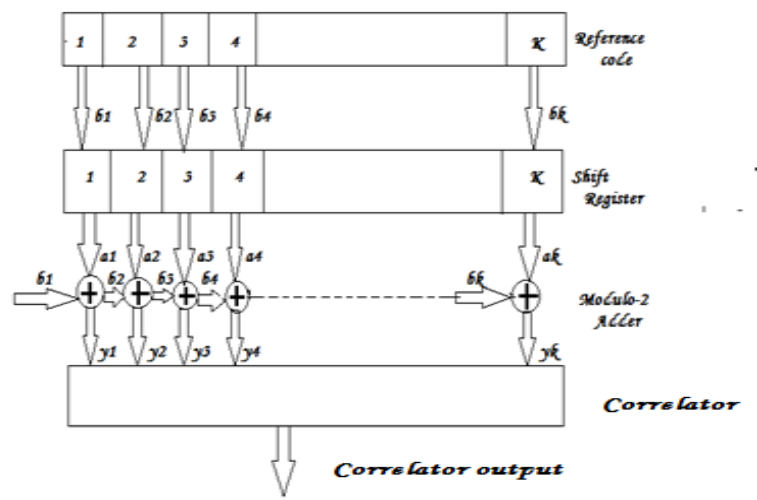

Figure 6 : A Correlator.
Fig.2 shows a correlator of length $K$. One sequence is $a_{i}$ shifted through a K-bit shift register and the output of each stage is applied to a set of $K$, XNOR gates for comparison. The other inputs of the XNOR gates receive the second sequence, $b_{i}$ The output of an XNOR gate working as a modulo- 2 adder is ' 1 ' if both inputs are the same and ' 0 ' otherwise. Summing these outputs in a K-input adder gives the correlation output, which may be divided by $K$ to get a normalised value. When the sequences $a_{i}$ and $b_{i}$ are different the sum, $\sum y_{i}$ is a cross correlation, otherwise it is an autocorrelation. When the PN sequence is auto correlated we get $\sum y_{i}=K$ when both sequences are in-phase. When there is a shift of even one bit in either direction the PN sequence does not match with its shifted version and $y_{i}$ forms the same sequence with a different phase (recall the 'shift and add' property discussed earlier). For large $K$ the number of ' 0 's and ' 1 's is almost equal to $K / 2$, giving a normalised correlation of $1 / 2$. This is true even for one sequence length, $L=2^{N}-1$. Thus, a PN sequence has an autocorrelation of 1 at zero phase (no time shift), and 0.5 at all other phases. The discrete autocorrelation values obtained for a PN sequence of length $2^{4}-1$ is shown in Fig. 7. At zero shift, and shifts which are multiples of 15 bits, the correlation value is 15 ; for all other shifts in either direction it is 7. This property is used in the synchronisation, or acquisition, of PN sequences.

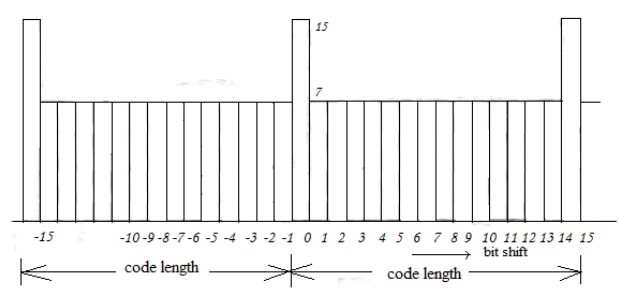

Figure 7 : Discrete auto-correlation of a PN sequence of length The auto-correlation can be used to predict the start of a PN sequence by detecting the peak. For an ideal PN sequence, its autocorrelation is two-valued and is given by the function:

$$
R_{a}(0)=L \text { and } R_{a}(k)=0 \text { for } 1 \leq k \leq L-1 .
$$

\section{CIRCUIT DIAGRAM}

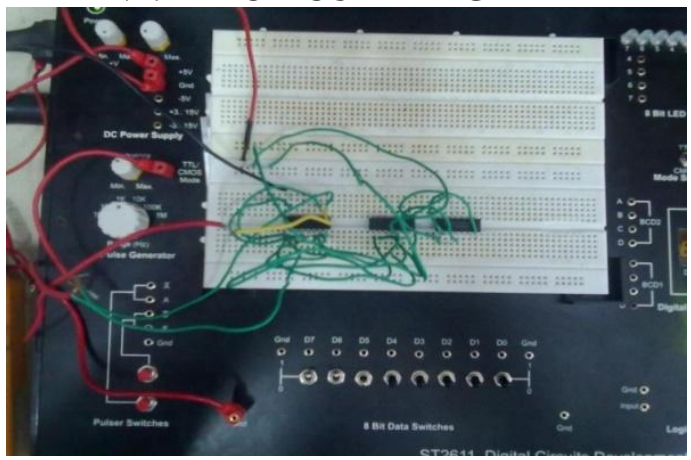

Figure 8: Circuit diagram for the PN sequence generator 
VII. RESULTING OUTPUT

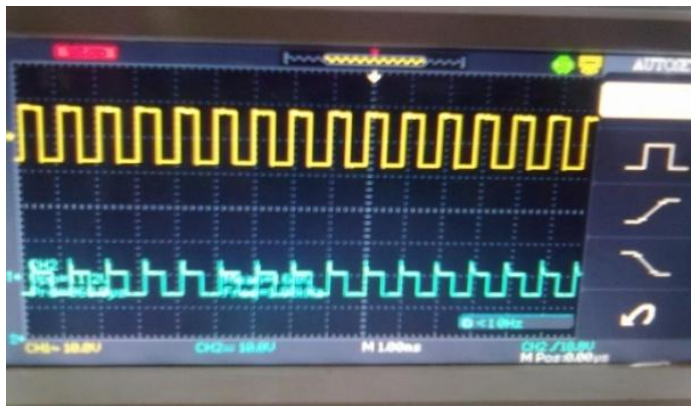

Figure 9 : Experimental Output of Auto-Correlation properties of $\mathrm{PN}$ sequence

\section{RESULTING ANALYSIS IN CROSS CORRELATION}

Both in Signal and Systems analysis, the concept of autocorrelation and cross correlation play an important role in Pseudo Noise sequences.. In the following sections, we present some the waveform analysis how these two functions may be estimated in Matlab for this random (Pseudo) generated noise sequences.

For sampled signal (i.e. sampled signal), the autocorrelation is defined as either biased or unbiased defined as follows :

$R_{X X}(m)=\frac{1}{N-|m|} \sum_{n=1}^{N-m+1} x(n) x(n+m-1)$ for biased

Autocorrelation

$R_{X X}(m)=\frac{1}{N} \sum_{n=1}^{N-m+1} x(n) x(n+m-1)$ for unbiased

Autocorrelation

The cross correlation function however measures the dependence of the values of one signal on another signal. For two WSS (Wide Sense Stationary) processes $\mathrm{x}(\mathrm{t})$ and $\mathrm{y}(\mathrm{t})$ it is described $R_{X Y}(t)=\lim _{\tau \rightarrow \infty} \frac{1}{T} \int_{-T}^{T} x(t) y(t+\tau) d t$ $R_{Y X}(t)=\lim _{\tau \rightarrow \infty} \frac{1}{T} \int_{-T}^{T} y(t) x(t+\tau) d t$

Now the following figure shows the effects of corresponding waveforms one by ones using autocorrelation as well as cross correlation process :
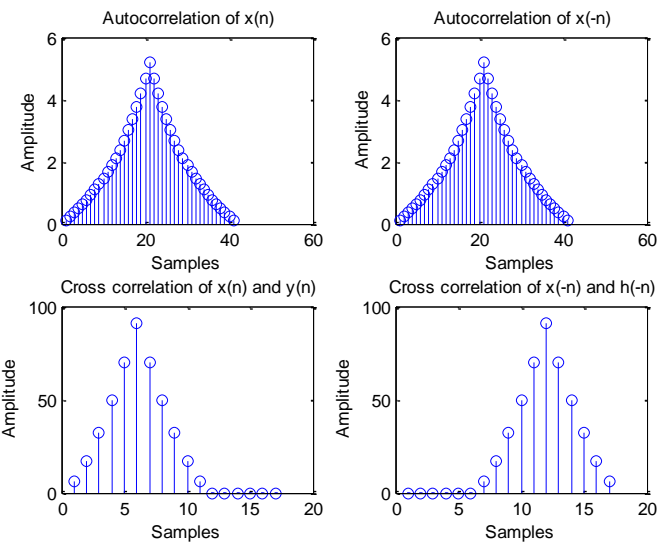

Figure 10 : Autoorrelation and Cross correlation in discrete time systems
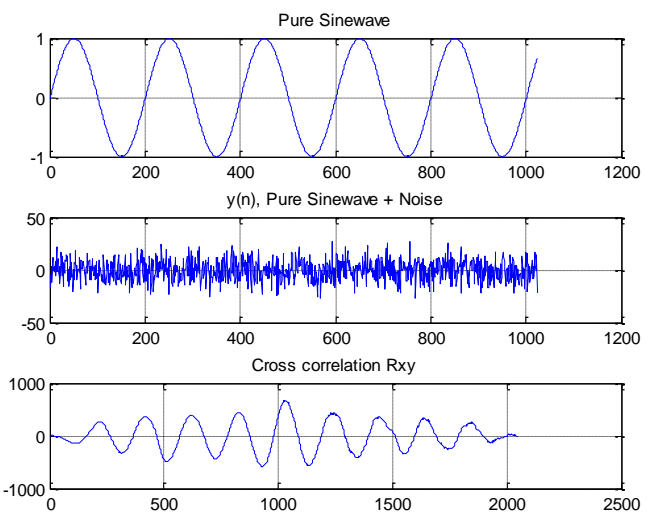

Figure 11 : Effects of Cross correlation in sine wave

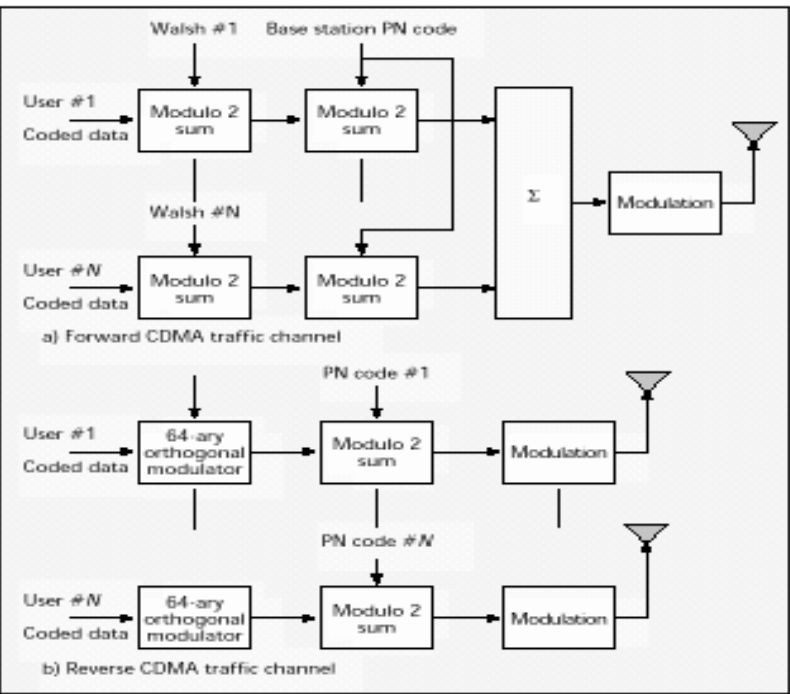

Figure 12 : Application of Walsh functions and PN codes in the forward and reverse links of cellular CDMA

\section{CONCLUSION}

Now on the basis of Pseudo Binary random signals case if we consider the continuous domain signals then the corresponding effects of cross correlation in sine wave analysis is shown in Fig.11. The middle portion shown the distorted part of the sinusoidal waveform. Now the Pseudo Noise signal in digital analysis will modulate the orthogonal functions into the information stream of the CDMA signal shown in Fig. 12

\section{ACKNOWLEDGMENTS}

The authors would like to thank the authorities of Birbhum Institute of Engineering and Technology for providing every kind of supports and encouragement during the working process. The authors thanks to reviewers for giving us such attention and time. The authors also acknowledge the unknown referees for their valuable comments and suggestions for improvement. Last but not the least the authors are giving a vote of thanks to our nearest and dearest parents and our be loving family members for providing mentally support to us.

\section{REFERENCES}

[1] Alka Sawlikar, Manisha Sharma, "Analysis of Different Pseudo Noise Sequences", IJCTEE Volume 1, Issue 2, ISSN 2249-6343.

[2] Afaq Ahamad,Sayyid Samir Al-Busaidi and Mufeed Juma AlMusharafi,"O properties of PN Sequences Generated by LFSR a 
Generalized Study and Simulation Modeling",IJST Volume.6(10),October 2013,print ISSN:0974-6846.

[3] Mutagi, RN "Pseudo Noise Sequences for Engineers", Electronics and Communication Engineering Journal, April 1996, IEE 1996

[4] Meghna Sharma, Rajeev Mathur,"A Method to Generation and Simulation of PN Sequence in MATLAB”, IJETAE, Volume2, Issue 7, July 2012, ISSN 2250-2459.

[5] Haykin.S \& Van Veen.B, 'Signals And Systems', $2^{\text {nd }}$ Edition, New York: John Wiley and Sons Inc, 1999, ISBN: 0-471-13820-7.

[6] Haykin. S, 'Communication System'. $4^{\text {th }}$ Edition. New York: John Wiley and Sons Inc, 2001, ISBN: 0-471-17869-1.

[7] Santit Traithavil," Simulation of PN Code Sequences for Cellular systems “,Department of Engineering, The Australian National University, February 7, 2006.

[8] J.G. Proakis,'Digital Communications'. $4^{\text {th }}$ Edition. United States: McGraw-Hill, 2000, ISBN-0-07-118183-0.

[9] Lathi, B.P, 'Modern Digital and Analog Communications System'. $3^{\text {rd }}$ Edition, New York: Oxford University Press, 1998, ISBN 019564801-3.

[10] P. K. Gupta, R. Kumarsen "Binary Multiplication with PN Sequences" IEEE Transactions on Acoustics Speech and Signal Processing. Vol. 36, no. 4, pp. 603- 605, 2005.

[11] Golome, S.W.: 'Shift register sequences' (Holden Day, San Francisco, CA, USA, 1967).

[12] Dixon, R.C.: 'Spread spectrum systems' (John Wiley, 1984).

\section{BIOGRAPHIES}

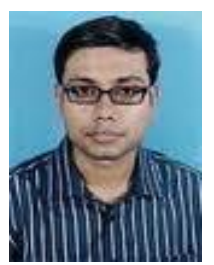

Nirmalya Chandra was born in India. He has been an Assistant Professor in the Department of Electronics and Communication Engineering, Birbhum Institute of Engineering and Technology ( Govt. Aided Institution ), Suri, Birbhum731101. His currently research interest in Control System, Adhoc Networks , Digital Modulation, Digital Signal Processing etc.

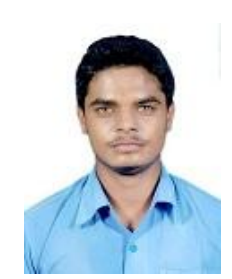

Sandeep Dey was born in India. He is a student in the Department of Electronics and Communication Engineering, Birbhum Institute of Engineering and Technology ( Govt. Aided Institution ), Suri, Birbhum-731101 under West Bengal University of Technology, Kolkat since 2011. His currently research interest in Signals and Systems, Digital Modulation, Digital Signal Processing etc.

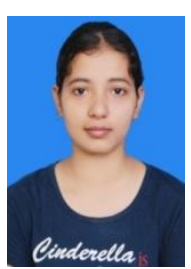

Sweta Shankar was born in India. She is pursuing B.Tech in Electronics and Communication Engineering from Birbhum Institute of Engineering \& Technology, Suri Affiliated to West Bengal University of Technology, Kolkata since 2011. Her main research interests are Digital Signal Processing and Digital Communication.

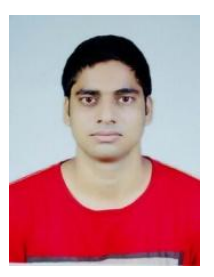

Roushan kumar was born in India. He is pursuing B.Tech in Electronics and Communication Engineering from Birbhum Institute of Engineering \& Technology, Suri Affiliated to West Bengal University of Technology, Kolkata since 2011. His main research interests are Digital Signal Processing and Digital Communication. 